\title{
Communication Needs of Elderly at Risk of Falls and their Remote Family
}

\author{
Corina Sas \\ Lancaster University \\ Lancaster, UK \\ corina@comp.lancs.ac.uk \\ Kieran Brahney \\ Lancaster University \\ LA1 4WA, UK \\ k.brahney@lancaster.ac.uk \\ Carl Oechsner \\ Lancaster University \\ LA1 4WA, UK \\ c.oechsner@lancaster.ac.uk \\ Amish Trivedi \\ Lancaster University \\ LA1 4WA, UK \\ a.trivedi@lancaster.ac.uk \\ Zaffar Mughal \\ Lancaster University \\ LA1 4WA, UK \\ z.mughal@lancaster.ac.uk \\ Keith Cheverst \\ Lancaster University \\ Lancaster, UK \\ k.cheverst@lancaster.ac.uk \\ Sarah Clinch \\ Lancaster University \\ Lancaster, UK \\ s.clinch@lancaster.ac.uk

\section{Nigel Davies} \\ Lancaster University \\ Lancaster, UK \\ n.a.davies@lancaster.ac.uk \\ Mauricio Nomesque \\ Lancaster University \\ LA1 4WA, UK \\ m.nomesquesilva@lancaster.ac.uk
}

Paste the appropriate copyright/license statement here. ACM now supports three different publication options:

- ACM copyright: ACM holds the copyright on the work. This is the historical approach.

- License: The author(s) retain copyright, but ACM receives an exclusive publication license.

- Open Access: The author(s) wish to pay for the work to be open access. The additional fee must be paid to ACM.

This text field is large enough to hold the appropriate release statement assuming it is single-spaced in Verdana 7 point font. Please do not change the size of this text box.

Each submission will be assigned a unique DOI string to be included here.

\section{Abstract}

The aging population experiences increased health risks, both physical and emotional. Two such risks are those of isolation and falling. This papers draws from $\mathrm{HCI}$ literature in these two independent research areas to explore the needs of family communication with elderly parents at risk of falls. We report on a study with 7 elderly parents and 3 of adult children, as well as a group interview with 12 elderly living in a sheltered accommodation. Findings indicate important emotional needs on both parts: adult children's anxiety for the wellbeing of their parents at risk of falls, and elderly's need for autonomy and their appreciation for an aesthetic design. We concluded with implications of these findings for designing for family communication in this challenging context.

\section{Author Keywords}

Fall detection, family communication needs, elderly people

\section{ACM Classification Keywords}

H.5.m. Information interfaces and presentation (e.g., $\mathrm{HCI}$ ): Miscellaneous. 


\section{Introduction}

With the global trend of increased aging population, and the higher cost of healthcare, support for elderly people's independent living becomes increasingly important. In addition, their connectedness with their adult children is also vital for avoiding isolation. A specific health risk for elderly people is that of falling, with $28 \%$ to $35 \%$ of people aged 65 and over falling every year [43]. In addition, the number of injuries caused by falls is anticipated to be $100 \%$ higher by 2030 . Unsurprisingly, research into fall detection technologies has gained momentum over the last decade. A parallel research direction regarding elderly's risk of isolation has focused on communication technologies for supporting remote connectedness with their adult children and grandchildren. This paper aims to intersect these largely independent research areas to explore the communication needs of elderly at risk of falls and their remote families. The study addresses the following research questions:

(i) What are adult children's needs for communication with their elderly parents at risk of falls? Do they prefer automatic notifications that everything is OK?

(ii) What are elderly's needs for communication with their remote family? Do they experience fear of falls, or they value more their autonomy?

(iii) What is elderly's attitude towards fall detection technology? Do they accept or resist its adoption?

\section{Related Work}

The emerging trend of ageing in place and provision of healthcare into the home [14] has fostered a growing interest in ambient assistive living and self-monitoring. Such work has spanned research areas from supporting memory $[10,34,39,40]$ and mental health [33] to assistance in the end of life care [35] or supporting the needs of the caregivers $[5,19]$. HCI research focusing on designing for elderly people $[24,25]$ and their specific needs (including those of caregivers) has identified several challenges around the issues of privacy $[6,18,43]$ and control $[1,7,17,41]$. Our study draws from $\mathrm{HCI}$ interest in technologies for fall detection and work on novel technologies [32] for remote family communication with elderly relatives.

Fall Detection Technologies

In the last two decades there has been an increased interest in automatic monitoring technologies for detecting elderly people's falls. El-Bendary and colleagues [12] have reviewed academic work on fall detection technologies between 2003 and 2013. They identified a range of fall monitoring systems from video surveillance and sensor networks, to mobile technologies. Some mobile devices involve body-worn sensors located on the wrist, waist or around one's neck [1]. Degen and colleagues [11] implemented a wrist-watch connected to a call center, augmented with tri-axial accelerometers. Marquis-Faulkes and colleagues [26] developed a waterproof wrist watch for elderly people in a sheltered home. The research prototype has been followed by a commercial solution integrating smartwatch with smartphone to detect the fall of elderly people affected by dementia. Besides tracking a person's movement, such body-worn devices can be augmented with biosensors to measure blood pressure or heart rate [20].

Previous work has highlighted two key requirements for fall detection technologies such as unobtrusiveness and durability. For example, Marquis-Faulkes [26] and Gövercin [16] found that elderly people would be most happy with unobtrusive devices. This need has been linked to devices' being bulky and therefore perceived 
by elderly people as stigmatizing [23]. As falls can occur in different places, including showers, devices should also be durable and waterproof [26]. Other relevant work is the integrated caregiver-focused mHealth framework providing fall detection services to elderly people [15]. The system integrates mobile phones with smartwatches and neural network algorithms to classify fall events. While the emphasis is on providing a rich set of contextual information about the elderly person's lifestyle, the fall notification is not detailed, and the paper presents limited information about user's input into its design. Another example is Arcelus and colleagues' fall detection system [2] which informs relatives but the paper does not provide details for its mechanics.

To conclude, most of such prior work has focused primarily on fall detection and less on fall notification. Notification has been mostly addressed through interfaces for either activating the alarm, or for turning off false alarms [37]. There has been also limited work exploring the emotional needs associated with increased risk of falls that both elderly people and their remote family members have in this context. This is surprising, giving that the risk of falls is high among elderly people and potentially a source of anxiety for the rest of the family living remotely. For this, we turn to the HCI literature on remote communication.

\section{Remote Family Communication}

Most HCI research on family communication has taken place within CSCW community, highlighting people's preference for synchronous communication $[4,22,29]$. With increased aging population, more extended families face the challenge of keeping in touch and managing the risks associated with elderly people's independent living. Van Bel and his colleagues [38] identified five dimensions of social connectedness consisting of shared understanding, mutual awareness of other's experiences, feeling of closeness, the quality of the contact, and saliency of the relationship. Mynatt and colleagues [27] proposed a three-pronged approach for designing technologies to extend elderly's independent living by mitigating crisis, supporting daily routines, and offering peace of mind for adult children. For example, they designed digital family portraits for capturing and visualizing a remote elderly parent's daily life, with the aim of supporting peace of mind for their adult children $[28,31]$. While the design has not intended to alert the family when the elderly's health is poor, it could potentially be extended to include such notifications.

A related system is CareNet Display [9] which provides ambient interactive access of local members of an elderly's care network to the elderly person's day-today unexpected events across seven key areas such as meals, medication, activities, mood, and falls.

Empowering elderly people to decide which information to be shared has emerged as particularly important. Another example is the family window system, a video communication tool utilizing always-on video $[7,9]$. This was intended to support feelings of connectedness within the remote family, in particular through communication between adult children and elderly parents. More subtle forms of social awareness among remote family members have been explored through systems such as SnowGlobe [42]; a lamp which glows when movement is identified in the remote living room. This system suggests the value of ambiguous and physical interaction. Additional work has focused on ambient awareness of routines for expressing emotions between older adults and family member in order to ensure family connectedness $[4,8,30]$. 
To summarise, much work has focused on sensing technologies for capturing and communicating behavioural and emotional responses to the remote family members $[2,3,26]$. Social awareness is often ensured through asynchronous channels in the form of tangibles and displays for historic or real-time data. While most work has focused on sharing and experiencing positive emotions about daily life, there has been less work into designing for family communication in challenging situations where the need for connectedness is intertwined with the need to manage anxiety around the risk of falls.

\section{Method}

The aim of this study is to explore the design space for family technologies for automatically detecting and communicating fall events of elderly users living independently or in sheltered accommodation. The study involved semi-structured interviews with seven elderly people, three living independently and four living in sheltered accommodation; and three adult children. In addition to these individual interviews, we also run a group interview with 12 elderly people in a sheltered accommodation. The recruitment process involved emails sent to several local sheltered accommodations and following one positive reply, we visited the accommodation and recruited participants in person. Most of the interviews took place face to face, and a few by phone. All interviews were recorded and transcribed.

Questions concerned elderlies' experience of falls, the concerns triggered by falls and their relevance for both the elderly people and their family members. We also asked about technology's potential to regularly inform family members of one's wellbeing, and elderly people's attitude towards monitoring: "If you were to fall, would you like a device to contact relatives or emergency services?"; "Would you be happy with the device contacting people automatically?"

Interviews with family members focused on the frequency of contact with their elderly relative and the preferred communications methods. Additional questions probed the potential of fall monitoring technology such as form of interaction, frequency and time of use, information provided, as well as: "Would you like a device that tracks her movements all day and instantly informs you if she fell down?"

Data analysis consisted of a hybrid approach where existing concepts such as dimensions of connectedness [38], autonomy and peace of mind [27] were used for the deductive coding, while new concepts grounded on the empirical data contributed to the inductive coding [12]. The codes were iteratively refined as new codes emerged under the themes of family's anxiety, elderly's conflicting needs for autonomy and privacy on the one hand, and safety on the other hand, and special communication needs derived from these.

\section{Findings}

We now describe each theme, illustrating it with quotes form participants' answers, i.e. C1-3 for children, and P1-7 for elderly parent. The three themes capture emotional aspects: anxiety of adult children, autonomy of the elderly parent, and the aesthetics design of such technologies.

Family's Anxiety for Elderly Parent at Risk of Falls Anxiety was a major theme throughout the family members' interviews. This is an important finding which goes beyond family's concerns for the wellbeing of their elderly relative $[27,28]$ : "You do wonder sometimes, like last night there was an ambulance that went past 
and you think is it mother again [...] "is she okay"? I mean, we were cross with Mum when she fell out of bed and she didn't press the buzzer because she didn't want to bother anybody. Something to alert us when something has happened and whether she was okay or not [would be useful]" [C2]. As shown above, the risk of falls is perceived as high by the adult children and with negative consequences for the parent's life. As a result, communication with their parents is regular consisting of daily phone calls and weekly visits.

Several participants mentioned that whilst they do this because they are anxious, they also enjoy talking and seeing their elderly parent.

When asked about automatic notifications such as " $I$ am well" or "everything is OK" participants expressed interest, albeit they have different views with respect to the prefer frequency of such notifications. Some adult children would prefer to check at their convenience, while others would want daily automatic notifications "as often the parent wants to say" all well" as she doesn't want me to worry or rush around. Likewise, notification of a fall is always required immediately as it means the difference between life and death" [C1].

With respect to the content of notification, some participants wanted rich information such as medical data, of contact of an available neighbour, whilst others expressed severe concerns that such medical

information could increase their anxiety. An important findings was that such monitoring device should provide needed reassurance that their elderly parent was okay but that it should only be used as an aid and not reduce the current communication methods and in particular the personal, face to face interaction. This suggests that the need of connectedness between the family members still needs to be accounted for [29].
Autonomy and Privacy of Elderly People at Risk of Falls In sharp contrast with the anxiety of their adult children, elderly parents appear to value more their autonomy, particularly if they are well enough to live independently: "I would find [a fall detection device] invasive. [...] Sometimes I fall over and I'm quite alright [so] I don't want anybody fussing around me. And also if my son knew I was falling over, then I'll be in serious trouble. That's why I personally would find it intrusive because I like to be as independent as possible" [P6].

This finding highlights a tension between the peace of mind that such technology will provide to the children, and the demand for autonomy and subsequently privacy of the elderly parent [10]. Interestingly, even after they have experienced falls, some elderly people do not seem to experience fear: "I have had two falls recently [because] I actually passed out. [But] I'm not really afraid because $I$ don't sort of think about" [P3].

This quote illustrates that anxiety is experienced mostly by those in charge with the caregiving, i.e., adult children, and that parents at risk of falls may benefit from being sensitised towards the value of prompt fall notifications. For example, elderly participants acknowledging that their children are worried about them falling, shown increased interest in wearing such devices for tracking and communicating falls.

Most of the interviewed elderly people made little use of such technology and tend to resist it. A different pattern emerged in the case of elderly people in the sheltered accommodation who tended to wear the panic button wrapped around their neck for manually triggering alarms to a call center. 
Aesthetics of Fall Detection Devices

An interesting finding was elderly's interest in the aesthetics of fall detection devices. Particularly those who were using the "panic button" were explicit that the device should be unobtrusive: "on the wrist would be best, something like a watch. I wouldn't want the device to be too big, such that it becomes not a very good sight to be - I want it to be discrete" [P2]. Other participants expressed interest in a device which is not only discrete but also aesthetically pleasing: "It would be preferable if the design was nice rather than naff. That's why I had [the panic button around my neck so it can be hidden under my blouse" [P1]. In addition, all participants in the group interview have agreed that "the device should have a modern design if possible".

\section{Implications for Design}

Study findings suggest three design implications [36] extending previous findings on the importance of negotiating between the value of continual contact and one's need for autonomy [21], within the context of risks of falls.

Lowering Adult Children's Anxiety for Elderly Parents The anxiety experienced by adult children for their elderly parents at risk of falls suggests the value of augmenting traditional fall monitoring devices with communication abilities to send regular daily automatic notifications signalling "all is well". This can be used to complement additional voice or face to face interactions, rather than substitute them.

Negotiating Children's Anxiety and Elderly's Autonomy Despite the risks that serious falls entail, some elderly people living independently expressed a strong sense of autonomy. Others conceded to fall tracking and communication devices, aware that it may bring peace of mind to their children. This suggests that such devices should be configurable, providing control to the elderly person with respect to easily setting the content and frequency of notifications. In order to be adopted, such devices should be discrete and aesthetically pleasing to avoid stigmatization.

Communication Needs in the Context of Risks of Falls Findings indicate also more subtle issues around elderly's control of what is being communicated, to whom, and the tracking of a diverse range of spatiotemporal contexts within and outside their home. Elderly people's communication needs include preventing false alarms, customizing the list of family members to whom fall notifications are automatically sent, and having their falls detected in a range of contexts from showering and sleeping, to working in the garden. Communication needs of the remote family members include real time access to the elderly people's status, as captured by the fall tracking devices. An additional communication channel should be made available to the family members to discretely nudge their loved ones for example if a longer period of inactivity is detected: "all is well?". This could ensure that their loved one is alright by actively sending them a ping which they have to answer to.

\section{Conclusion}

This paper explored communication needs between elderly parents at risk of falls and their adult children living remotely. We report on interviews with 19 elderly and 3 adult children. The findings indicate three emotional needs centred on elderly's autonomy and value for aesthetic design, and adult children's anxiety for the wellbeing of their parents at risk of falls. We concluded with three implications for design addressing these emotional needs. 


\section{References}

1. Aarhus Rikke and Stinne Aaløkke Ballegaard. 2010. Negotiating boundaries: managing disease at home. In Proceedings of the Conference on Human Factors in Computing Systems, 1223-1232.

2. Amaya Arcelus, Megan Howell Jones, Rafik Goubran, and Frank Knoefel. 2007. Integration of smart home technologies in a health monitoring system for the elderly. In Advanced Information Networking and Applications Workshops, 820-825.

3. Automated Security Alerts. 2012. Retrieved December 16, 2016 at: http://automatedsecurityalert.com/fallguard-pro/

4. Margot Brereton, Alessandro Soro, Kate Vaisutis, and Paul Roe. 2015. The Messaging Kettle: Prototyping Connection over a Distance between Adult Children and Older Parents. In Proceedings of the Conference on Human Factors in Computing Systems (CHI '15). ACM, New York, 713-716.

5. Jed Brubaker, Gina Venolia and John Tang. 2012. Focusing on shared experiences: moving beyond the camera in video communication. Proc. of the Designing Interactive Systems Conference, 96-105.

6. Kelly Caine, Arthur D. Fisk, and Wendy A. Rogers. 2006. Benefits and privacy concerns of a home equipped with a visual sensing system: A perspective from older adults. Proc. Human factors and ergonomics society, 50,2: 180-4.

7. Yunan Chen, Victor Ngo, and Sun Young Park. 2013. Caring for caregivers: designing for integrality. In Proceedings of the Conference on Computer supported cooperative work, 91-102.

8. Raymundo Cornejo, Mónica Tentori and Jesús Favela. 2013. Ambient awareness to strengthen the family social network of older adults. Computer Supported Cooperative Work, 22.2-3: 309-344.

9. Sunny Consolvo, Peter Roessler, and Brett E. Shelton. 2004. The CareNet display: lessons learned from an in home evaluation of an ambient display. International Conference on Ubiquitous Computing. Springer Berlin Heidelberg, 1-17.

10. Nigel Davies, Adrian Friday, Sarah Clinch, Corina Sas, Marc Langheinrich, Geoff Ward, G. and Albrecht Schmidt. 2015. Security and Privacy Implications of Pervasive Memory Augmentation. IEEE Pervasive Computing, 14, 1: 44-53.

11. Thomas Degen, Heinz Jaeckel, Michael Rufer and Stefen Wyss. 2003. SPEEDY: A Fall Detector in a Wrist Watch. In ISWC, 184-189.

12. Nashwa El-Bendar, Qing Tan, Frédérique C. Pivot, and Anthony Lam. 2013. Fall detection and prevention for the elderly: A review of trends and challenges. International Journal on Smart Sensing and Intelligent Systems, 6,3: 1230-1266.

13. Jennifer Fereday and Eimear Muir-Cochrane. 2006. Demonstrating rigor using thematic analysis: $A$ hybrid approach of inductive and deductive coding and theme development. International journal of qualitative methods, 5, 1: 80-92.

14. Geraldine Fitzpatrick and Gunnar Ellingsen. 2013. A review of 25 years of CSCW research in healthcare: contributions, challenges and future agendas. Computer Supported Cooperative Work, 22.4-6: 609-665.

15. Mohammed Ghazal, Yasmina Al Khalil, Fatemeh Jalil Dehbozorgi, and Marah Talal Alhalabi. 2015. An integrated caregiver-focused mHealth framework for 
elderly care. Wireless and Mobile Computing, Networking and Communications, 238-245.

16. Mehmet Gövercin, Költzsch, Y., Meis, M., Wegel, S., Gietzelt, M., Spehr, J., Winkelbach, S., Marschollek, M., and Steinhagen-Thiessen, E., 2010. Defining the user requirements for wearable and optical fall prediction and fall detection devices for home use. Informatics for health and social care, 35, 3-4: 177-187.

17. Erik Grönvall and Nervo Verdezoto. 2013. Beyond self-monitoring: understanding non-functional aspects of home-based healthcare technology. In Proceedings of the Conference on Pervasive and ubiquitous computing (UbiComp '13), 587-596.

18. Tsipi Heart and Efrat Kalderon. 2013. Older adults: are they ready to adopt health-related ICT? International journal of medical informatics 82,11 : 209-231.

19. Lesa Huber, Kalpana, Kelly Caine, Kay Connelly, L. Jean Camp, Beth Ann Walker and Lisa Borrero. 2013. How in-home technologies mediate caregiving relationships in later life. International Journal of Human-Computer Interaction 29,7: 441-55.

20. Jae Min Kang, Taiwoo Yoo and Hee Chan Kim 2006. A wrist-worn integrated health monitoring instrument with a tele-reporting device for telemedicine and telecare. IEEE Transactions on Instrumentation and Measurement, 55,5: 1655-1661.

21. Tejinder K. Judge, Carman Neustaedter, Steve Harrison, and Andrew Blose. 2011. Family portals: connecting families through a multifamily media space. In Proceedings of the SIGCHI Conference on Human Factors in Computing Systems (CHI '11). ACM, New York, NY, USA, 1205-1214
22. David Kirk, Abigail Sellen and Xiang Cao. 2010. Home Video Communication: Mediating "Closeness". In Proceedings of the conference on Computer supported cooperative work (CSCW), Seattle: ACM Press, 135-144.

23. Ilkka Korhonen, Juha Parkka, and Mark Van Gils. 2003. Health monitoring in the home of the future. Engineering in Medicine and Biology Magazine, IEEE, 22,3: 66-73.

24. Sian Lindley, Richard Harper and Abigail Sellen. 2008. Designing for elders: exploring the complexity of relationships in later life. In Proceedings of British HCI Conference, 77-86.

25. Sian Lindley, Richard Harper and Abigail Sellen. 2009. Desiring to be in touch in a changing communications landscape: attitudes of older adults. In: Proc. of the Conference on Human Factors in Computing Systems, 1693-1702.

26. Fran Marquis-Faulkes, Stephen McKenna, Alan Newell and Peter Gregor. 2005. Gathering the requirements for a fall monitor using drama and video with older people. Technology and Disability, 17(4), 227-236.

27. Elizabeth Mynatt, Irfan Essa and Wendy Rogers. 2000. Increasing the opportunities for aging in place. In Proceedings on the Conference on Universal Usability, 65-71. ACM

28. Elizabeth Mynatt, Jim Rowan, Sarah Craighill and Annie Jacobs. 2001. Digital family portraits: supporting peace of mind for extended family members. In Proceedings of the Conference on Human factors in computing systems, 333-340. ACM. 
29. Carman Neustaedter, Steve Harrison and Abigail Sellen. 2013. Connecting families: an introduction. In Connecting Families, 1-12. Springer London.

30. Yann Riche and Wendy Mackay. 2010. PeerCare: supporting awareness of rhythms and routines for better aging in place. Computer Supported Cooperative Work (CSCW) 19.1: 73-104.

31. Jim Rowan and Elizabeth D. Mynatt. 2005. Digital Family Portrait Field Trial: Support for Aging in Place. In Proc. CHI '05, 521-530.

32. Antti Salovaara, Kristina Höök, Keith Cheverst, Michael Twidale, Matthew Chalmers, and Corina Sas. 2011. Appropriation and creative use: linking user studies and design. In CHI '11 Extended Abst. 37-40.

33. Corina Sas and Rohit Chopra, R. 2015. MeditAid: A Wearable Adaptive Neurofeedback-based System for Training Mindfulness State. Personal and Ubiquitous Computing, 19,7: 1169-1182.

34. Corina Sas, Tomasz Fratczak, Matthew Rees, Hans Gellersen, Vaiva Kalnikaite, Alina Coman, and Kristina Höök. 2013. AffectCam: arousalaugmented Sensecam for richer recall of episodic memories. In CHI '13 Extended Abstracts, 10411046.

35. Corina Sas, Shuang Ren, Alina Coman, Sarah Clinch, and Nigel Davies. 2016. Life Review in End of Life Care: A Practitioner's Perspective. In CHI Extended Abstracts, 2947-2953

36. Corina Sas, Steve Whittaker, Steven Dow, Jodi Forlizzi, and John Zimmerman. 2014. Generating implications for design through design research. In Proceedings of the 32nd annual ACM conference on
Human factors in computing systems ( $\mathrm{CHI}$ '14). ACM, New York, NY, USA, 1971-1980.

37. Jonathan Tomkun and Binh Nguyen. (2010). Design of a fall detection and prevention system for the elderly. Retrieved December 19, 2016 from: https://macsphere.mcmaster.ca/handle/11375/14451

38. Daniel van Bel, Smolders, K. C., Wijnand A. IJsselsteijn and Yvonne A.W. de Kort, Y. 2009. Social connectedness: concept and measurement. In Intelligent Environments 20,2: 67-74.

39. Elise van Den Hoven, Corina Sas and Steve Whittaker. 2012. Introduction to this special issue on designing for personal memories: past, present, and future. Human-Computer Interaction, 27,1-2: 1-12.

40. Huy Viet Le, Sarah Clinch, Corina Sas, Tilman Dingler, Niels Henze, and Nigel Davies. 2016. Impact of Video Summary Viewing on Episodic Memory Recall: Design Guidelines for Video Summarizations. In Proc. CHI '16, 4793-4805

41. John Vines, Stephen Lindsay, Gary W. Pritchard, Mabel Lie, David Greathead, Patrick Olivier, and Katie Brittain. 2013. Making family care work: dependence, privacy and remote home monitoring telecare systems. In Proc. UbiComp '13, 607-616.

42. Thomas Visser, Martijn H. Vastenburg, and David V. Keyson. 2011. Designing to support social connectedness: The case of SnowGlobe. International Journal of Design 5, 3: 129-142.

43. Katherine Wild, Linda Boise, Jay Lundell, and Anna Foucek. 2008. Unobtrusive in-home monitoring of cognitive and physical health: Reactions and perceptions of older adults. Journal of Applied Gerontology, 27 2: 181-200. 